\title{
On the Kinematics of Vehicles Relevant to Conflict Detection and Resolution
}

\author{
Luís Braga Campos ${ }^{1}$ and Joaquim Guerreiro Marques $\mathbb{D}^{1,2}$ \\ ${ }^{1}$ CCTAE (Center for Aeronautical and Space Science and Technology), IDMEC/LAETA, Instituto Superior Técnico, Universidade de \\ Lisboa, Av. Rovisco Pais 1, 1049-001 Lisboa, Portugal \\ ${ }^{2}$ Escola Náutica Infante D. Henrique, Av. Engenheiro Bonneville Franco, 2770-058 Paço de Arcos, Oeiras, Portugal \\ Correspondence should be addressed to Joaquim Guerreiro Marques; jmgmarques@tecnico.ulisboa.pt
}

Received 17 April 2018; Accepted 11 July 2018; Published 19 August 2018

Academic Editor: Jose E. Naranjo

Copyright (c) 2018 Luís Braga Campos and Joaquim Guerreiro Marques. This is an open access article distributed under the Creative Commons Attribution License, which permits unrestricted use, distribution, and reproduction in any medium, provided the original work is properly cited.

\begin{abstract}
The paper presents methods to determine the time, positions, and distance of closest approach for two vehicles following arbitrary trajectories in two or three dimensions. The distance of closest approach of two vehicles following arbitrary curved trajectories is determined by two conditions: (i) the relative velocity must be orthogonal to the relative position in order for the distance to be a nonzero extremum; (ii) the radial acceleration including centripetal terms must have a direction that increases the separation for the extremum to be a minimum. This theorem on the distance of closest approach simplifies in the case of uniform motion along rectilinear trajectories. Three examples are given: (i) the two-dimensional motion of surface vehicles changing the velocity of one of them so as to enforce a given minimum separation distance; (ii) the three-dimensional motion of two aircraft, one flying horizontally and the other climbing, changing the vertical velocity of the latter to ensure a minimum separation distance set "a priori"; (iii) the case of an aircraft flying with constant velocity in a straight line so that its closest approach to another aircraft flying in a circular holding pattern in the same plane occurs at a given time chosen "a priori".
\end{abstract}

\section{Introduction}

In the traffic of vehicles safety is identified with the absence of collisions or conflicts. A conflict occurs when the distance between the centroids of two vehicles is less than a safe separation distance (SSD) determined by their size. Thus (i) the absence of conflicts and (ii) the confirmation that a conflict has been resolved depend on determining the distance of closest approach (DCA) that is not less than the SSD. The conflict resolution relies (iii) on trajectory modifications that change the DCA from smaller than the SSD to larger than (or equal to) the SSD. The paper presents methods to determine the time, positions, and distance of closest approach for two vehicles following arbitrary trajectories in two or three dimensions. The two-dimensional cases include cars in road traffic, ships in sea lanes, and aircraft ground movements at an airport. The three-dimensional cases include all types of flying vehicles, like airplanes, helicopters, drones, rockets and satellites, and also submerged submarines. The differences in conflict detection and resolution (CDR) between all these types of vehicles concern the speed, size, and distances that enter as parameters in the same methods of calculation of distance and time of closest approach.

The distance and time of closest approach are essential inputs for CDR methods [1-3]. The collision risk applies to cars $[4,5]$, ships and submarines [6,7], and aircraft $[8,9]$. Taking as example the case of Air Traffic Management (ATM) the problem may be divided into (i) prediction of flight paths $[10,11]$, (ii) safety assessment $[12,13]$, and (iii) conflict resolution [14-16]. Collision avoidance between aircraft starts with [17] separation distances (e.g., longitudinal, lateral, and altitude) leading to high Target Level of Safety (TLS: probability of collision less than $5 \times 10^{-9}$ per hour) for various aircraft encounter geometries, like level crossing [18] or climb and descent [19]. The safe separation ultimately determines airspace capacity [20]. Many of these methods assume straight trajectories or approximate curved trajectories by straight segments. While this allows a continuous 
trajectory, the velocity becomes discontinuous in direction at the edges and the acceleration becomes singular. The purpose of the present paper is to determine the distance and time of closest approach for arbitrary curved trajectories without approximations of any kind. This may be used for CDR methods or to assess TLS.

There are a variety of CDR methods including multiagent algorithms [21-23] that apply to vehicles moving in two [19, 24-27] or three [10-20] dimensions. All CDR methods (i) start with the identification of a conflict, (ii) involve trajectory changes to resolve the conflict, and (iii) end with the verification that the conflict has been resolved. The safety of traffic requires that a minimum SSD be held, for example, ensuring that the "safety volumes" around two vehicles do not penetrate. If two vehicles follow two arbitrary trajectories with position vectors $\vec{x}_{1}(t)$ and $\vec{x}_{2}(t)$, as a function of time, the modulus of the difference specifies their distance that generally varies with time:

$$
D(t)=\left|\vec{x}_{1}(t)-\vec{x}_{2}(t)\right|
$$

A conflict is detected if an time the distance is less than the SSD:

$$
\text { Conflict: } D(t)<\delta \quad \text { for some time } t \text {. }
$$

If a conflict is detected, then one or both trajectories must be modified to resolve the conflict. The success of the conflict resolution is checked by showing that the distance exceeds the SSD for all time:

$$
\text { No Conflict: } D(t) \geq \delta \text { for all time } t \text {. }
$$

The criteria for conflict (2) or no conflict (3) are next put into a simpler form that is easier to apply.

The key concept is that of distance of closest approach $D_{*}$ between two trajectories:

$$
D_{*} \equiv D\left(t_{*}\right)=\left|\vec{x}_{1}\left(t_{*}\right)-\vec{x}_{2}\left(t_{*}\right)\right|
$$

which occurs at the time $t_{*}$ of closest approach, when the vehicles are at position $\vec{x}_{1}\left(t_{*}\right)$ and $\vec{x}_{2}\left(t_{*}\right)$. There is a conflict if the distance of closest approach is less than the safe separation distance (5a) and no conflict otherwise (5b):

$$
\begin{aligned}
& D_{*}<\delta \text { conflict, } \\
& D_{*} \geq \delta \text { no conflict. }
\end{aligned}
$$

The paper presents a method to determine (i) the distance of closest approach $D_{*}$, (ii) the time of closest approach $t_{*}$, and (iii) the positions of the two vehicles at that time $\vec{x}_{1}\left(t_{*}\right), \vec{x}_{2}\left(t_{*}\right)$. The method applies to arbitrary trajectories: (a) curved or rectilinear; (b) with constant velocity, accelerated or decelerated motion. The method is deterministic and excludes external disturbances. The extension to include random disturbances can be made adding to the position vectors the deviations due to uncertainties or external effects and applying statistical methods.

Considering first arbitrary nonuniform motion a theorem is established (Section 2.1) specifying the conditions for minimum separation between two trajectories. In many traffic situations the future trajectories are not known, and the information available is only the current positions and velocities of two vehicles; if the motion is assumed to be uniform, simple formulas are obtained (Section 2.2) for the distance and time of closest approach. This in turn specifies the conditions for collision avoidance between two or any number of vehicles (Section 2.3). These conditions give a simple geometric interpretation of the two theorems (Section 2). The preceding theory is applied (Section 3) to three examples: (Section 3.1) two-dimensional collision avoidance between surface vehicles (ships, car, or airplanes on the ground at an airport) with constant velocity by choosing the modulus of the velocity of one of them; (Section 3.2) three-dimensional collision avoidance between two aircraft moving at constant velocity, one at constant altitude and the other climbing, by changing the vertical velocity of the latter; (Section 3.3) meeting a given time of closest approach between a vehicle in a holding pattern of uniform circular motion and another in uniform rectilinear motion. These three cases are sufficiently simple for analytical calculation (Section 3), illustrate the two theorems (Section 2), and substantiate the discussion (Section 4).

\section{Distance of Closest Approach between Trajectories}

The minimal separation for two vehicles following arbitrary trajectories is obtained by minimizing the relative distance as a function of time. This leads to two conditions to be satisfied at the time of closest approach (Section 2.1). The simplest case is that of uniform motion from given initial positions (Section 2.2). It leads to a simple criterion for collision avoidance by maintaining a minimum separation distance (Section 2.3) and is applicable to two or more vehicles. The vehicles are represented by material vehicles placed at their centroids, and their dimensions are taken into account by setting a safe separation distance to avoid collisions.

2.1. Nonuniform Motion along Curved Trajectories. Consider two vehicles moving with integrable time-dependent velocities $\vec{V}_{1}(t)$ and $\vec{V}_{2}(t)$ from initial positions $\vec{x}_{10}$ and $\vec{x}_{20}$ at time $t=0$, so that their positions at time $t$ are given by

$$
\begin{aligned}
& \vec{x}_{1}(t)=\vec{x}_{10}+\int_{0}^{t} \vec{V}_{1}(t) d t \\
& \vec{x}_{2}(t)=\vec{x}_{20}+\int_{0}^{t} \vec{V}_{2}(t) d t .
\end{aligned}
$$

It is necessary to find (i) the conditions for a collision, where the positions coincide with $\vec{x}_{1}\left(t_{*}\right)=\vec{x}_{2}\left(t_{*}\right)$ for some time(s) $t=t_{*}$; (ii) if there is no collision then the time $t=t_{*}$ and distance $D_{*}$ of closest approach are given by $(4) \equiv(7)$ :

$$
\begin{aligned}
D_{*} & \equiv D\left(t_{*}\right)=\left|\vec{x}_{1}\left(t_{*}\right)-\vec{x}_{2}\left(t_{*}\right)\right| \\
& =\left|\vec{x}_{1}(t)-\vec{x}_{2}(t)\right|_{\min } .
\end{aligned}
$$


The relative position of the two vehicles is given at all times by:

$$
\begin{aligned}
\vec{x}(t) & \equiv \vec{x}_{1}(t)-\vec{x}_{2}(t) \\
& =\vec{x}_{10}-\vec{x}_{20}+\int_{0}^{t}\left[\vec{V}_{1}(t)-\vec{V}_{2}(t)\right] d t .
\end{aligned}
$$

Introducing the initial relative positions (9a) at the initial time $t=0$ and the relative velocity (9b) at all times,

$$
\begin{aligned}
\vec{x}_{0} & \equiv \vec{x}_{10}-\vec{x}_{20}, \\
\vec{V}(t) & \equiv \vec{V}_{1}(t)-\vec{V}_{2}(t),
\end{aligned}
$$

the relative position at arbitrary time is given by

$$
\vec{x}(t)=\vec{x}_{0}+\int_{0}^{t} \vec{V}(t) d t=\vec{x}_{0}+\vec{X}
$$

where the change in relative position from time $t=0$ to time $t$ is given by (1la):

$$
\begin{aligned}
\vec{X}(t) & =\int_{0}^{t} \vec{V}(t) d t, \\
\frac{d \vec{X}}{d t} & =\vec{V},
\end{aligned}
$$

and its time derivative is relative velocity (11b).

The distance between the vehicles at time $t$ is given by

$$
\begin{aligned}
{[D(t)]^{2} } & =|\vec{x}(t)|^{2}=\left|\vec{x}_{0}+\vec{X}(t)\right|^{2} \\
& =\left|x_{0}\right|^{2}+|\vec{X}(t)|^{2}+2 \vec{x}_{0} \cdot \vec{X}(t) .
\end{aligned}
$$

The first derivative of (12) with regard to time,

$$
\begin{aligned}
2 D(t) \frac{d D}{d t} & =\frac{d}{d t}[D(t)]^{2}=2\left[\vec{X}(t)+x_{0}\right] \cdot \frac{d \vec{X}}{d t} \\
& =2 \vec{x} \cdot \vec{V},
\end{aligned}
$$

is zero for stationary distance between the vehicles:

$$
\begin{aligned}
D^{\prime}\left(t_{*}\right) & =0 \Longrightarrow \\
0 & =\vec{x}\left(t_{*}\right) \cdot \vec{V}\left(t_{*}\right) .
\end{aligned}
$$

Thus two cases may arise: (i) if the distance is zero at the stationary point(s) then there is collision between the vehicles and the relative velocity is arbitrary:

$$
\begin{aligned}
& D\left(t_{*}\right)=0 \Longrightarrow \\
& \vec{x}\left(t_{*}\right)=0 ;
\end{aligned}
$$

(ii) if the distance is not zero then the velocity and relative position must be orthogonal:

$$
\begin{gathered}
D\left(t_{*}\right) \neq 0 \Longrightarrow \\
\vec{V}\left(t_{*}\right) \perp \vec{x}\left(t_{*}\right) .
\end{gathered}
$$

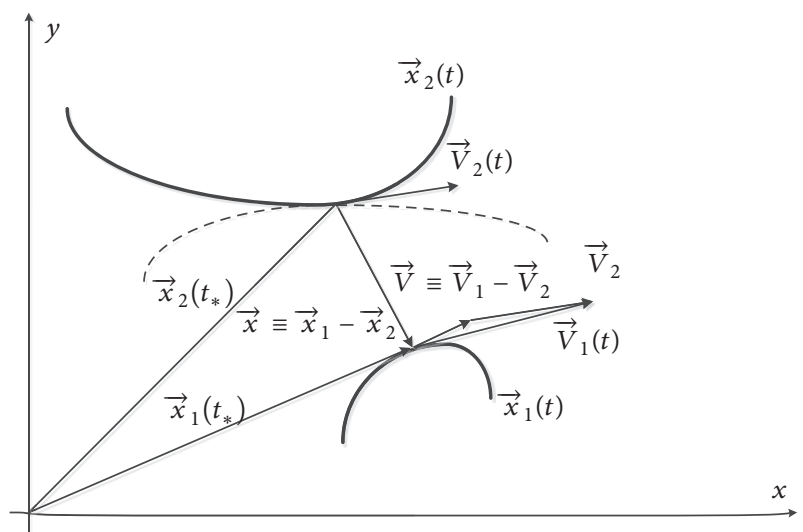

Figure 1: Two vehicles move along trajectories specified by the position vectors $\vec{x}_{1}(t)$ and $\vec{x}_{2}(t)$ as a function of time, so that the relative position is $\vec{x} \equiv \vec{x}_{1}-\vec{x}_{2}$. The trajectories may be curved and described in nonuniform motion so that the velocities are functions of time $\vec{V}_{1}(t)$ and $\vec{V}_{2}(t)$ as also the relative velocity $\vec{V} \equiv \vec{V}_{1}-\vec{V}_{2}$. The time of closest approach $t_{*}$ corresponds to relative position $\vec{x}$ orthogonal $\vec{x} \perp \vec{V}$ to the relative velocity $\vec{V}$; that is, their inner product is zero $\vec{x} \cdot \vec{V}=0$. The dotted line refers to a trajectory of the second vehicle also leading to an extremum of the relative distance but a maximum rather than a minimum.

Note that the distance of closest approach is generally not the smallest distance between the paths because the two vehicles will be at these points at different times. The condition of closest approach that the relative velocity and relative position are orthogonal (Figure 1) can be explained as follows: (i) if $\vec{V} \cdot \vec{x}<0$ at time $t=t_{*}$ then the vehicles would be moving towards each other and would be closer at some later time $t>t_{*}$; (ii) if $\vec{V} \cdot \vec{x}>0$ at time $t=t_{*}$ then the vehicles would be moving away from each other and would have been closer for some earlier time $t<t_{*}$. In either case (i) or (ii) the time $t=t_{*}$ would not be that of stationary distance. This proves by "reductio ad absurdum" that a stationary distance requires that the relative velocity be orthogonal to the relative position.

A stationary distance is a necessary but not sufficient condition for closest approach; an inflexion point in one of the trajectories would also correspond to a stationary distance but might not be a minimum; a minimum is determined by the second-order derivative of distance with regard to time:

$$
\begin{aligned}
2 D & (t) \frac{d^{2} D}{d t^{2}}+2\left(\frac{d D}{d t}\right)^{2}=\frac{d^{2}}{d t^{2}}\left\{[D(t)]^{2}\right\} \\
& =2 \frac{d}{d t}(\vec{x} \cdot \vec{V})=2\left(\vec{V} \cdot \vec{V}+\vec{x} \cdot \frac{d \vec{V}}{d t}\right),
\end{aligned}
$$

being positive at the stationary condition. In the stationary condition $(14 a) \equiv(18 a)$ the relation (17) simplifies to (18b):

$$
D^{\prime}\left(t_{*}\right)=0 \text { : }
$$

$$
2 D\left(t_{*}\right) D^{\prime \prime}\left(t_{*}\right)=2\left\{\left|\vec{V}\left(t_{*}\right)\right|^{2}+\vec{x}\left(t_{*}\right) \cdot \vec{a}\left(t_{*}\right)\right\}
$$

$$
>0 \text {, }
$$




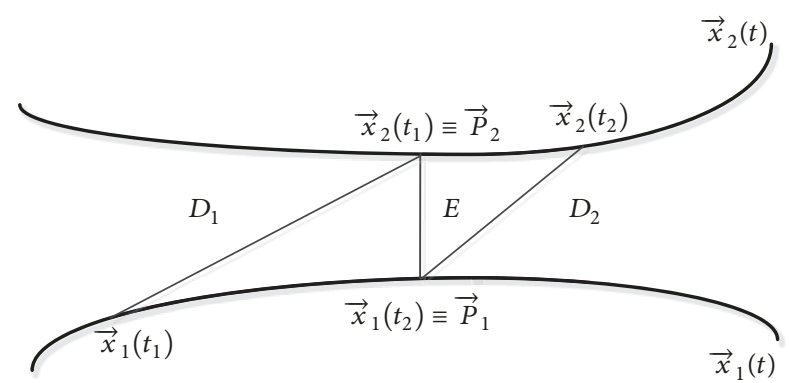

Figure 2: The shortest distance $E$ between two curves or paths is along the common normal. This would be the distance of closest approach $D$ along the two trajectories only if the two vehicles were at the closest positions $\left(P_{1}, P_{2}\right)$ at the same time. Generally the two vehicles are at the closest positions $\left(P_{1}, P_{2}\right)$ at different times $t \neq t_{1}$, so their distances $\left(D_{1}, D_{2}\right)$ are larger than $E$.

where (19a) is the relative acceleration that must satisfy (19b) for a minimum:

$$
\begin{array}{r}
\vec{a} \equiv \frac{\overrightarrow{d V}}{d t} \equiv \frac{d^{2} \vec{x}}{d t^{2}}: \\
\vec{x}\left(t_{*}\right) \cdot \vec{a}\left(t_{*}\right)>-\left|\vec{V}\left(t_{*}\right)\right|^{2} .
\end{array}
$$

Thus the stationary distance will be a minimum if the acceleration is along the relative position $\vec{a} \cdot \vec{x}>0$, because it tends to move the two vehicles away from each other. Even if the relative acceleration is from one vehicle towards the other $\vec{a} \cdot \vec{x}<0$, the distance will still be a minimum if the centripetal acceleration is larger:

$$
\begin{aligned}
& \vec{a} \cdot \bar{x}=-|\vec{a} \cdot \vec{x}|<0: \\
& \frac{|\vec{a} \cdot \bar{x}|}{|\vec{x}|}<\frac{|\vec{V}|^{2}}{|\vec{x}|} .
\end{aligned}
$$

The stationary point would not be one of closest approach only if the relative acceleration projected on the relative position is opposite in sign and larger in modulus than the centripetal acceleration.

The preceding results may be summarized in a theorem on nonuniform motion: two vehicles with initial positions $\vec{x}_{10}$ and $\vec{x}_{20}$ and nonuniform velocities, respectively, $\vec{V}_{1}$ and $\vec{V}_{2}$, which are integrable functions of time, will collide at an arbitrary angle if their relative distance ((8), (12)) vanishes ((15a), (15b)). If the vehicles do not collide, (i) the distance is stationary, which is a necessary condition for closest approach, if and only if ((16a), (16b)) the relative velocity (9b) is orthogonal to the relative position (8) इ(10); (ii) a sufficient condition for closest approach, which is that the distance is minimum, is that the relative acceleration (19a) satisfies (19b) implying that the centripetal acceleration associated with the relative curvature of the trajectories predominates over the relative acceleration projected on the relative velocity.

The distance of closest approach $D$ between vehicles moving two trajectories generally exceeds the smallest distance

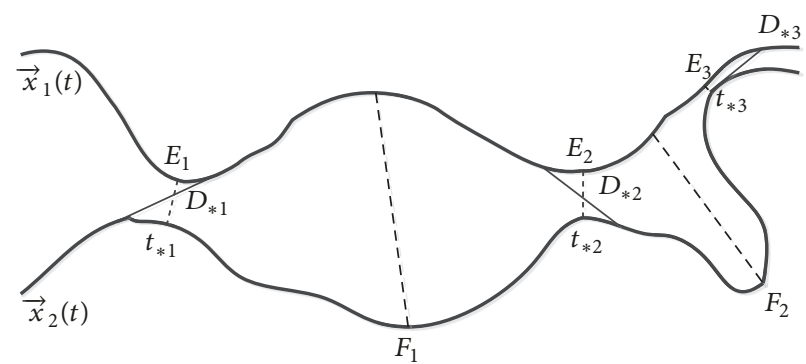

FIGURE 3: For vehicles moving along curved trajectories there may exist several times $t_{*_{n}}$ of closest approach $D_{*_{n}}$, corresponding to local minima of the relative distance that usually exceeds the minimum distances $E_{n}$. The distance of closest approach is then the smallest of all local minima $D_{*_{n}}$. There may also exist local maxima $F_{n}$ indicated by dotted lines.

$E$ between the two paths, because the vehicles might cross the closest points at different times; for example (Figure 2), (i) at the time $t_{1}$ when vehicle 2 is at the closest position $\vec{x}_{2}\left(t_{1}\right)=\vec{P}_{2}$ vehicle 1 is still behind the closest position $\vec{x}_{1}\left(t_{2}\right) \neq \vec{P}_{1}$ :

$$
D_{1} \geq E \leq D_{2} ;
$$

(ii) by the time $t_{2}$ vehicle 1 reaches the closest position $\vec{x}_{2}\left(t_{2}\right)=\vec{P}_{1}$ vehicle 2 will be beyond the closest position $\vec{x}_{2}\left(t_{2}\right) \neq \vec{P}_{2}$. Conditions (16b) and (18b) of closest approach are local, in the sense that for small deviations to earlier or later time the relative distance increases. In the case of curved trajectories there can exist several times $t_{*_{n}}$, distances $D_{*_{n}}$, and positions $\vec{x}_{1}\left(t_{*_{n}}\right), \vec{x}_{2}\left(t_{*_{n}}\right)$ of local closest approach as shown in Figure 3. In this case the distance of closest approach would be the "minimum minimorum" that is the smallest or infimum of all local distances of closest approach:

$$
D_{*}=\inf \left(D_{*_{1}}, D_{*_{2}}, \ldots, D_{*_{n}}\right) .
$$

The existence of multiple local minima of the distance is not possible for straight trajectories: if the two vehicles move with constant velocity there is only one time and distance of closest approach, as shown next.

2.2. Closest Approach for Uniform Rectilinear Motion. In many traffic situations the future trajectories of vehicles are not known or can be adjusted to avoid collisions. The information available from traffic sensors is usually positions and velocities at a given time. The simplest assumption is that the velocities will be constant and the position of the centroids of the vehicles, taken as material vehicles, at time $t$ will be

$$
\begin{aligned}
& \vec{x}_{1}(t)=\vec{x}_{10}+\vec{V}_{1} t, \\
& \vec{x}_{2}(t)=\vec{x}_{20}+\vec{V}_{2} t .
\end{aligned}
$$

The relative position at time $t$ is

$$
\vec{x}(t) \equiv \vec{x}_{1}(t)-\vec{x}_{2}(t)=\vec{x}_{0}+\vec{V} t,
$$


where $(9 a)$ is the initial relative position and the relative velocity $(9 \mathrm{~b})$ is constant. The relative distance at time $t$ is given by

$$
\begin{aligned}
{[D(t)]^{2} } & =|\vec{x}(t)|^{2}=\left|\vec{x}_{0}+\vec{V} t\right|^{2} \\
& =\left|\vec{x}_{0}\right|^{2}+|\vec{V}|^{2} t^{2}+2 t \vec{x}_{0} \cdot \vec{V}
\end{aligned}
$$

The second-order derivative with regard to time is always positive:

$$
\frac{d^{2}}{d t^{2}}\left\{[D(t)]^{2}\right\}=2|\vec{V}|^{2}>0
$$

and thus a stationary distance can only be a minimum distance. There is no maximum distance because a constant relative velocity leads the vehicles infinitely apart after a long time.

The first derivative of the distance

$$
2 D(t) \frac{d D}{d t}=\frac{d}{d t}\left\{[D(t)]^{2}\right\}=2\left(\vec{x}_{0} \cdot \vec{V}+|\vec{V}|^{2} t\right)
$$

vanishes at the time of closest approach:

$$
\begin{aligned}
D(t) & \geq|D(t)|_{\min }=D\left(t_{*}\right): \\
t_{*} & =-\frac{\vec{V} \cdot \vec{x}_{0}}{|\vec{V}|^{2}}=-\frac{\left|\vec{x}_{0}\right|}{|\vec{V}|} \cos \alpha
\end{aligned}
$$

where $\alpha$ is the angle of the relative initial position (9a) with the constant relative velocity (9b). The position of the two vehicles at the time of closest approach is obtained by substituting (28b) in (23a), (23b):

$$
\begin{aligned}
& \vec{x}_{1}\left(t_{*}\right)=\vec{x}_{10}-\frac{\vec{V}_{1}}{|\vec{V}|^{2}} \cdot\left(\vec{V} \cdot \vec{x}_{0}\right), \\
& \vec{x}_{2}\left(t_{*}\right)=\vec{x}_{20}-\frac{\vec{V}_{2}}{|\vec{V}|^{2}} \cdot\left(\vec{V} \cdot \vec{x}_{0}\right),
\end{aligned}
$$

and their relative position is given by

$$
\begin{aligned}
\vec{x}\left(t_{*}\right)= & \vec{x}_{1}\left(t_{*}\right)-\vec{x}_{2}\left(t_{*}\right) \\
= & \vec{x}_{10}-\vec{x}_{20}-\frac{\left(\vec{V} \cdot \vec{x}_{0}\right)}{|\vec{V}|^{2}}\left(\vec{V}_{1}-\vec{V}_{2}\right) \\
= & \vec{x}_{0}-\frac{\vec{V}}{|\vec{V}|^{2}}\left(\vec{V} \cdot \vec{x}_{0}\right) \\
= & \frac{\vec{x}_{0}|\vec{V}|^{2}-\vec{V}\left(\vec{V} \cdot \vec{x}_{0}\right)}{|\vec{V}|^{2}}=\frac{\vec{V} \wedge\left(\vec{x}_{0} \wedge \vec{V}\right)}{|\vec{V}|^{2}},
\end{aligned}
$$

where the double outer product of vectors was used.
The distance of closest approach (31b) is the modulus of the relative position vector $(30) \equiv(31 \mathrm{a})$ :

$$
\begin{aligned}
& \vec{x}\left(t_{*}\right)=\frac{\vec{V} \wedge\left(\vec{x}_{0} \wedge \vec{V}\right)}{|\vec{V}|^{2}}, \\
& D_{\text {min }}=D\left(t_{*}\right)=\frac{\left|\vec{V} \wedge\left(\vec{x}_{0} \wedge \vec{V}\right)\right|}{|\vec{V}|^{2}} .
\end{aligned}
$$

Denoting by $\vec{N}$ the unit vector orthogonal to the plane of the initial relative position (9a) and constant relative velocity (9b) leads to the following: (i) the outer product (32a):

$$
\begin{array}{r}
\vec{x}_{0} \wedge \vec{V}=\left|\vec{x}_{0}\right||\vec{V}| \sin \alpha \vec{N} \\
\left|\vec{V} \wedge\left(\vec{x}_{0} \wedge \vec{V}\right)\right|=\left|\vec{x}_{0}\right||\vec{V}|^{2} \sin \alpha
\end{array}
$$

(ii) since the outer product involving $\vec{V}$ is orthogonal to $\vec{V}$, it follows (32b). Substituting (32b) in (31b) specifies the distance of closest approach (33):

$$
D_{\min }=\left|\vec{x}_{0}\right| \sin \alpha>\delta
$$

which must exceed the safety distance to avoid a conflict between the vehicles. The distance of closest approach can also be obtained substituting the time of closest approach (28b) in the distance (25):

$$
\begin{aligned}
\left(D_{\text {min }}\right)^{2} & =\left[D\left(t_{*}\right)\right]^{2} \\
& =\left|\vec{x}_{0}\right|^{2}+|\vec{V}|^{2}\left[\frac{\vec{x}_{0} \cdot \vec{V}}{|\vec{V}|^{2}}\right]^{2}-2 \frac{\left(\vec{x}_{0} \cdot \vec{V}\right)^{2}}{|\vec{V}|^{2}} \\
& =\left|\vec{x}_{0}\right|^{2}-\frac{\left(\vec{x}_{0} \cdot \vec{V}\right)^{2}}{|\vec{V}|^{2}}=\left|\vec{x}_{0}\right|^{2}\left(1-\cos ^{2} \alpha\right) \\
& =\left|\vec{x}_{0}\right|^{2} \sin ^{2} \alpha,
\end{aligned}
$$

in agreement with (33).

2.3. Collision Avoidance for Several Vehicles. The preceding results with uniform motion apply to collision avoidance with two vehicles, as stated in the theorem on uniform motion: two vehicles with given distinct initial positions $\left(\vec{x}_{10}, \vec{x}_{20}\right)$ and constant velocities $\left(\vec{V}_{1}, \vec{V}_{2}\right)$ collide (35a) only if the initial 


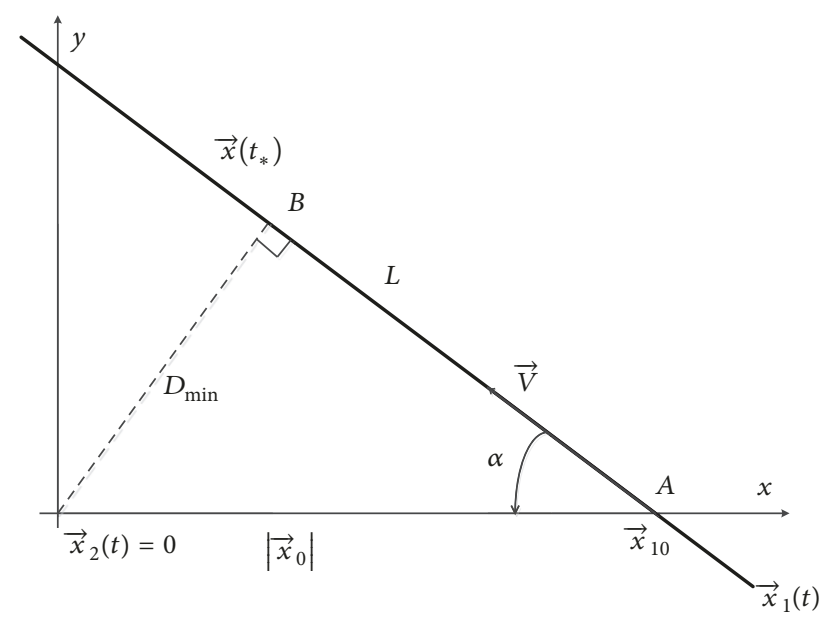

Figure 4: The distance of closest approach $D_{m}$ is a unique minimum in the case of constant velocities when the trajectories are straight lines described in uniform motion. The distance of closest approach in this case is calculated most simply by choosing a Cartesian reference frame moving with the velocity of the second vehicle, so that it stays at the origin $\vec{x}_{2}(t)=0$ for all time. The OX axis is taken through the initial position $\vec{x}_{10}$ of the first vehicle, at a distance $\left|\vec{x}_{10}\right|$. The trajectory of the first vehicle is a straight line in the direction of the relative velocity making an angle $\alpha$ with the $x$ axis. The distance of the closest approach is thus (33). The distance covered by the first vehicle is (37a), and since it travels at the relative velocity $|V|$ the time of closest approach is the ratio (37b).

relative position (9a) and the constant relative velocity (9b) are antiparallel $(35 b) \equiv(35 c)$ :

$$
\begin{gathered}
D_{\min }=0: \\
\alpha=\pi, \\
\vec{x}_{0} \|-\vec{V}, \\
t_{*}=\frac{\left|\vec{x}_{0}\right|}{|\vec{V}|},
\end{gathered}
$$

in which case collision (28b) occurs at time (35d). If the relative velocity makes an angle $\alpha$ with the initial relative position, the time of closest approach is (28b) and the distance of closest approach is (33). In order that a minimum safety distance $\delta$ can be maintained at all times it is sufficient that the angle of the constant relative velocity (9b) with the relative initial position (9a) satisfies

$$
\sin \alpha>\frac{\delta}{\left|\vec{x}_{0}\right|}=\sin \alpha_{0}
$$

In this case one vehicle "misses the other" or "passes by" at a distance larger than the minimum separation distance.

These results can be interpreted by a simple geometrical construction involving elementary Euclidean geometry (Figure 4): (i) since only relative motion is of interest the origin $\mathrm{O}$ is taken at the second vehicle; (ii) the initial position $A$ of the first vehicle specifies the initial distance $\left|\vec{x}_{0}\right|$; (iii) through A a straight line is drawn in the direction of the constant relative velocity that makes an angle $\alpha$ with the relative initial position; (iv) an orthogonal line through the origin intersects at the point $\mathrm{B}$ of closest approach; (v) it follows that the distance of closet approach is (33); (vi) the distance travelled by the first vehicle from the initial position to the point of closest approach is (37a):

$$
\begin{aligned}
L & =\left|\vec{x}_{0}\right| \cos \alpha ; \\
-t_{*} & =\frac{L}{|\vec{V}|}=\frac{\left|\vec{x}_{0}\right|}{|\vec{V}|} \cos \alpha,
\end{aligned}
$$

(vii) since the relative velocity is constant the time of closest approach is (37b) in agreement with (28b); (viii) the minus sign arises because of the angle $\alpha$ is measured from the relative initial position to the constant relative velocity; (ix) at the point of closest approach the relative position (30) is orthogonal to the relative velocity:

$$
\vec{x}\left(t_{*}\right) \cdot \vec{V}=\frac{\vec{V} \cdot\left[\vec{V} \wedge\left(\vec{x}_{0} \wedge \vec{V}\right)\right]}{|\vec{V}|^{2}}=0,
$$

in agreement with the general condition ((16a), (16b)); (x) the condition (19b) for a minimum distance is met for zero relative acceleration $0>-|\vec{V}|^{2}$.

The angle $\alpha$ in (36) would be imaginary if $\sin \alpha>1$ but in this case $\left|\vec{x}_{0}\right|<\delta$ the initial separation would already be less than the minimum separation distance, and the safety criterion was violated from the start. If the safety condition is not violated from the start, the initial relative distance is larger than the safe separation distance, and a real angle $\alpha_{0}$ exists. It suffices that the angle of the relative velocity with the relative initial position exceeds this value in modulus $|\alpha|>\left|\alpha_{0}\right|$ for the safe separation to be ensured at all times. The problem of safe separation between two vehicles, with a given safe separation distance $\delta$ as a chosen parameter, may be extended to several vehicles, say $n+1$. Choosing vehicle 1 as the reference the exclusion of collision with each of the other $N$ vehicles leads to $N$ conditions of type (36). If these $N$ conditions are compatible the first vehicle can avoid a collision with all the others by being steered in a constant direction. If the $N$ conditions are incompatible a collision cannot be avoided in a straight path and a polygonal or curved path will be necessary. That may not be sufficient to avoid a collision if the traffic density is too high.

Three situations can arise concerning the time of closest approach (28b):

$$
\begin{array}{ll}
t_{*}>0 & \text { if } \vec{V} \cdot \vec{x}_{0}<0, \\
t_{*}=0 & \text { if } \vec{V} \cdot \vec{x}_{0}=0, \\
t_{*}<0 & \text { if } \vec{V} \cdot \vec{x}_{0}>0 .
\end{array}
$$

The time of closest approach is time zero (39b) if the initial relative position is orthogonal to the constant relative velocity, 


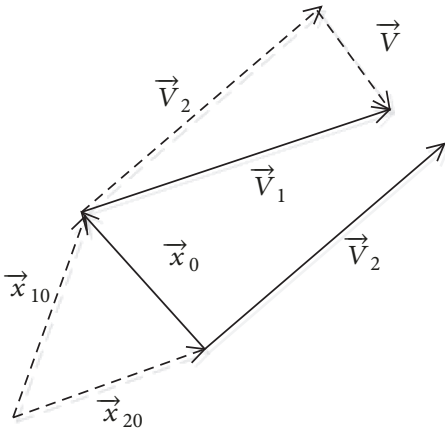

(a)

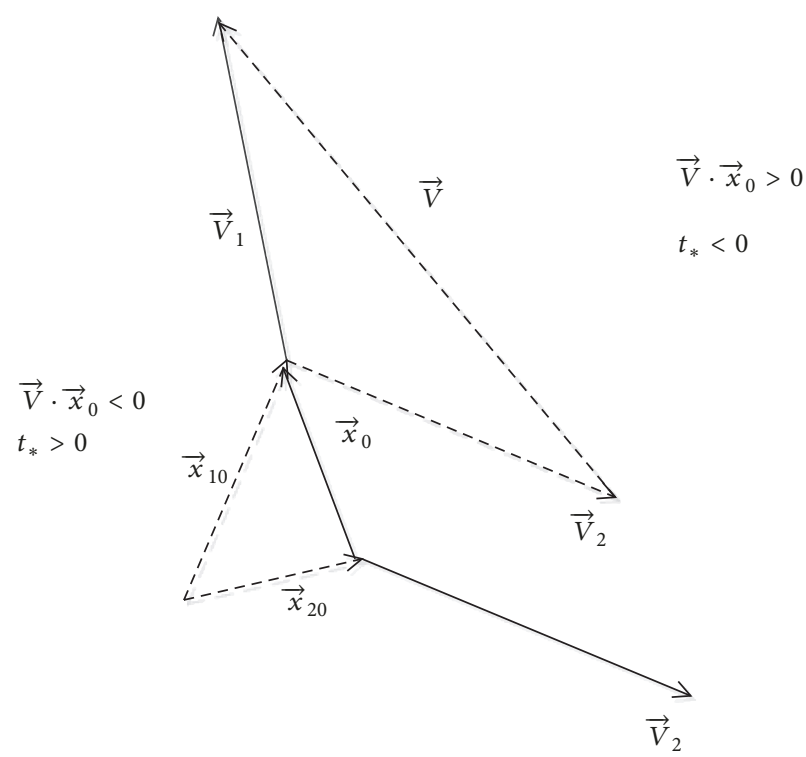

(b)

FIGURE 5: For vehicles moving with constant velocities $\left(V_{1}, V_{2}\right)$ if the relative velocity $\vec{V} \equiv \vec{V}_{1}-\vec{V}_{2}$ makes an obtuse angle $\vec{x}_{0} \cdot \vec{V}<0$ with the relative $\vec{x}_{0} \equiv \vec{x}_{10}-\vec{x}_{20}$ initial positions then (case (a)) the trajectories converge, and the time of closest approach is in the future $t_{*} \geq 0$. If the relative velocity $\vec{V}$ makes an acute angle $\vec{x}_{0} \cdot \vec{V}>0$ with the relative initial position $\vec{x}_{0}$, the trajectories diverge, and the closest approach was in the past $t_{*}<0$.

in agreement with condition (16b) of closest approach. If the vehicles move towards each other (39a) the time of closest approach is positive because (Figure 5(a)) the trajectories are converging and will be closest in the future. If the two vehicles are moving away from each other (39c) the time of closest approach is negative, because the trajectories are diverging (Figure 5(b)) and were closer in the past. Thus two situations arise: (a) for converging trajectories (40a), that is, positive time of closest approach (39a), the minimum separation distance is (31b):

$$
\begin{aligned}
& \vec{V} \cdot \vec{x}_{0}>0: \quad D_{\min }=|\vec{V}|^{-2}\left|\vec{V} \wedge\left(\vec{x}_{0} \wedge \vec{V}\right)\right|, \\
& \vec{V} \cdot \vec{x}_{0} \leq 0: \quad D_{\text {min }}=\left|\vec{x}_{0}\right| ;
\end{aligned}
$$

(b) for parallel or diverging trajectories (40b) the minimum separation distance is the initial separation, because the vehicles will not get closer in the future.

\section{Safe Separation between Straight and Curved Trajectories}

As examples of the preceding theory, the second theorem is used in the simplest case of uniform motion along straight paths (Sections 2.2 and 2.3) both in two (Section 3.1) and three (Section 3.2) dimensions motivated by collision avoidance between (i) ships or aircraft moving on ground at airports (Section 3.1)and (ii) aircraft in flight (Section 3.2). The first theorem is needed in the third (iii) case involving a curved path (Section 3.3).
3.1. Two-Dimensional Safe Separation between Ships in a Water. The first application is conflict avoidance between two ships; following nautical practice the speed is in knots (kt) in (41a) and heading (41b) in degrees:

$$
\begin{aligned}
& \left\{V_{1}, V_{2}\right\}=\{10,20\} k t, \\
& \left\{\varphi_{1}, \varphi_{2}\right\}=\left\{30^{\circ}, 150^{\circ}\right\},
\end{aligned}
$$

and hence time is in hours and distance in nautical miles $(\mathrm{nm})$, e.g., for the initial positions $(41 \mathrm{c}, \mathrm{d})$ in a Cartesian frame:

$$
\begin{aligned}
& \vec{x}_{10}=\{-10,5\} n m, \\
& \vec{x}_{20}=\{5,-15\} n m .
\end{aligned}
$$

It is necessary to find if the minimum separation distance satisfies the safety threshold of $20 \mathrm{~nm}$ :

$$
\left|\vec{x}_{1}(t)-\vec{x}_{2}(t)\right|_{\min } \geq \delta=20 \mathrm{~nm} ;
$$

if this is not the case then the modulus of the velocity of the second ship $V_{2}$ is to be modified to $V_{2}^{\prime}$ as little as possible so that the minimum safety distance is complied with. The problem would be similar with different values for the ground movements of two aircraft at an airport or other cases of twodimensional motion of vehicles like cars. 
The Cartesian components of the velocities of the two ships are

$$
\begin{aligned}
\left\{V_{1 x}, V_{1 y}\right\} & =V_{1}\left\{\cos \varphi_{1}, \sin \varphi_{1}\right\}=\{8.66,5.00\} k t \\
\left\{V_{2 x}, V_{2 y}\right\} & =V_{2}\left\{\cos \varphi_{2}, \sin \varphi_{2}\right\} \\
& =\{-17.32,10.00\} k t .
\end{aligned}
$$

This specifies the relative velocity (44a) and relative initial position (44b):

$$
\begin{aligned}
\vec{V} & =\vec{V}_{1}-\vec{V}_{2}=\{25.98,-5.00\} k t, \\
\vec{x}_{0} & =\vec{x}_{10}-\vec{x}_{20}=\{-15,20\} \mathrm{nm} .
\end{aligned}
$$

The angle between them is calculated from

$$
\begin{aligned}
|\vec{V}| & =26.46 \mathrm{kt}, \\
\left|\vec{x}_{0}\right| & =25 \mathrm{~nm}, \\
\cos \alpha & =\frac{\vec{V} \cdot \vec{x}_{0}}{|\vec{V}|\left|\vec{x}_{0}\right|}=-0.7403,
\end{aligned}
$$

where $(45 \mathrm{c})$ has two roots. chosen:

The angle is less than $180^{\circ}$ so the root (46a) of (45c) is

$$
\begin{aligned}
\alpha & =137.76^{\circ}, \\
D_{\text {min }} & =\left|\vec{x}_{0}\right| \sin \alpha=16.80 \mathrm{~nm}, \\
t_{*} & =-\frac{\left|\vec{x}_{0}\right|}{|\vec{V}|} \cos \alpha=0.6995 \mathrm{~h},
\end{aligned}
$$

leading to a distance of closest approach (46b) after a time (46c). At that time the position of the two ships is

$$
\begin{aligned}
& \vec{x}_{1}\left(t_{*}\right)=\vec{x}_{10}+\vec{V}_{1} \cdot t_{*}=\{-3.94,8.50\} n m, \\
& \vec{x}_{2}\left(t_{*}\right)=\vec{x}_{20}+\vec{V}_{2} \cdot t_{*}=\{-7.11,-8.00\} n m,
\end{aligned}
$$

confirming the separation $(46 \mathrm{~b}) \equiv(48 \mathrm{a})$

$$
\left|\vec{x}_{1}\left(t_{*}\right)-\vec{x}_{2}\left(t_{*}\right)\right|=16.80 \mathrm{~nm}<\delta \equiv 20 \mathrm{~nm},
$$

is less than the desired safe separation distance $(48 \mathrm{~b}) \equiv(42)$.

In the example shown the distance of minimal approach (46b) is less than the desired safe separation distance $(42) \equiv(48 \mathrm{~b})$, and thus the angle between the relative velocity and initial relative position must be decreased to $\alpha^{\prime}$ satisfying

$$
\begin{aligned}
\sin \alpha^{\prime} & =\frac{\delta}{\left|\vec{x}_{0}\right|}=0.8, \\
\alpha^{\prime} & =126.87^{\circ}, \\
\cos \alpha^{\prime} & =-0.6 .
\end{aligned}
$$

It is required that the desired minimum separation distance is to be obtained by changing only the modulus of velocity of the second ship from $V_{2}=20 k t$ in (41a) to $V_{2}^{\prime}$ to be determined next; the speed of the first ship $V_{1}$ is unchanged and the headings of the two ships $\left(\varphi_{1}, \varphi_{2}\right)$ are also maintained. The Cartesian components of the velocity of the first ship are the same (43a) and those of the second ship (43b) change to

$$
\left(V_{2 x}^{\prime}, V_{2 y}^{\prime}\right)=V_{2}^{\prime}\left\{\cos \varphi_{2}, \sin \varphi_{2}\right\}=\left\{-\frac{\sqrt{3}}{2}, \frac{1}{2}\right\} V_{2}^{\prime} .
$$

The relative initial position (44b) remains and the relative velocity (44a) changes to

$$
\vec{V}^{\prime}=\vec{V}_{1}-\vec{V}_{2}^{\prime}=\left\{5 \sqrt{3}+\frac{\sqrt{3}}{2} V_{2}^{\prime}, 5-\frac{1}{2} V_{2}^{\prime}\right\} .
$$

The square of the modulus of the relative velocity changes to

$$
\begin{aligned}
\left|\vec{V}^{\prime}\right|^{2} & =\left(5 \sqrt{3}+\frac{\sqrt{3}}{2} V_{2}^{\prime}\right)^{2}+\left(5-\frac{1}{2} V_{2}^{\prime}\right)^{2} \\
& =\left(V_{2}^{\prime}\right)^{2}+10 V_{2}^{\prime}+100 .
\end{aligned}
$$

The projection of the relative velocity (51) on the relative initial position (44b) changes to

$$
\begin{aligned}
\vec{V}^{\prime} \cdot \vec{x}_{0} & =-15\left(5 \sqrt{3}+\frac{\sqrt{3}}{2} V_{2}^{\prime}\right)+20\left(5-\frac{1}{2} V_{2}^{\prime}\right) \\
& =-29.90-22.99 V_{2}^{\prime} .
\end{aligned}
$$

Its square appears in (45c) modified to

$$
\left|\vec{V}^{\prime}\right|^{2}\left|\vec{x}_{0}\right|^{2} \cos ^{2} \alpha^{\prime}=\left(\vec{V}^{\prime} \cdot \vec{x}_{0}\right)^{2}
$$

Substitution of (52), (53), (45b), and (49c) in (54) leads to

$$
\begin{aligned}
225 & {\left[\left(V_{2}^{\prime}\right)^{2}+10 V_{2}^{\prime}+100\right] } \\
& =894+1374.8 V_{2}^{\prime}+528.5\left(V_{2}^{\prime}\right)^{2},
\end{aligned}
$$

which is a quadratic equation in $V_{2}^{\prime}$ :

$$
303.5\left(V_{2}^{\prime}\right)^{2}-875.2 V_{2}^{\prime}-21606=0,
$$

with roots:

$$
V_{2}^{\prime}=1.442 \pm 8.560
$$

The two roots,

$$
V_{2+}^{\prime}=10.002>V_{2-}^{\prime}=-7.118,
$$

correspond to slowing down $V_{2+}^{\prime}=10 k t<20 k t=V_{2}$ or reversing the motion $V_{2-}^{\prime}=-7.1 k t<0<20 k t=V_{2}$ of the second ship. They lead, respectively, to the relative velocities

$$
\begin{aligned}
& V_{+}^{\prime}=\left|\left(V_{1}\right)^{2}+\left(V_{2+}^{\prime}\right)^{2}-2 V_{1} V_{2+}^{\prime} \cos \alpha^{\prime}\right|^{1 / 2}=17.89 k t \\
& V_{-}^{\prime}=\left|\left(V_{1}\right)^{2}+\left(V_{2-}^{\prime}\right)^{2}-2 V_{1} V_{2-}^{\prime} \cos \alpha^{\prime}\right|^{1 / 2}=8.08 k t
\end{aligned}
$$


and distinct times of closet approach:

$$
\begin{aligned}
& t_{+}=-\frac{\left|\vec{x}_{0}\right|}{\left|\vec{V}_{+}^{\prime}\right|} \cos \alpha^{\prime}=0.8385 \mathrm{~h}, \\
& t_{-}=\frac{\left|\vec{x}_{0}\right|}{\left|\overrightarrow{V^{\prime}}\right|} \cos \alpha^{\prime}=-1.8564 \mathrm{~h} .
\end{aligned}
$$

The relative positions of the two ships at these times are

$$
\begin{aligned}
\vec{x}_{+} & =\vec{x}_{1}-\vec{x}_{2+}=\vec{x}_{10}+\vec{V}_{1} t_{+}-\vec{x}_{20}-\vec{V}_{2+}^{\prime} t_{+}=\vec{x}_{0} \\
& +\left(\vec{V}_{1}-\vec{V}_{2+}^{\prime}\right) t_{+}=\left\{x_{0}\right. \\
& +\left(V_{1 x}-V_{2+}^{\prime} \cos \varphi_{2}\right) t_{+}, y_{0} \\
& \left.+\left(V_{1 y}-V_{2+}^{\prime} \sin \varphi_{2}\right) t_{+}\right\}=\{-0.48,19.99\}, \\
\vec{x}_{-} & =\vec{x}_{1}-\vec{x}_{2-}=\vec{x}_{10}+\vec{V}_{1} t_{-}-\vec{x}_{20}-\vec{V}_{2-}^{\prime} t_{-}=\vec{x}_{0} \\
& +\left(\vec{V}_{1}-\vec{V}_{2-}^{\prime}\right) t_{-}=\left\{x_{0}\right. \\
& +\left(V_{1 x}-V_{2-}^{\prime} \cos \varphi_{2}\right) t_{-}, y_{0} \\
& \left.+\left(V_{1 y}-V_{2-}^{\prime} \sin \varphi_{2}\right) t_{-}\right\}=\{-19.63,4.11\} .
\end{aligned}
$$

Both solutions correspond to the desired minimum safety distance:

$$
\left|\vec{x}_{+}\right|=20.0 \mathrm{~km}=\delta=\left|\vec{x}_{-}\right| \text {, }
$$

obtained either by moving faster ahead (59a) or by slowing down (59b).

3.2. Three-Dimensional Safe Separation between Aircraft in Climb and at Cruise. The second example is conflict avoidance between aircraft in flight; following aeronautical practice the speed is in kilometers per hour, for the first aircraft in level flight (63a) and the second climbing (63b):

$$
\begin{aligned}
\vec{V}_{1} & =\{900,0,0\} \mathrm{km} / \mathrm{h}, \\
\vec{V}_{2} & =\{600,-200,50\} \mathrm{km} / \mathrm{h}, \\
\vec{x}_{10} & =\{0,0,10\} \mathrm{km}, \\
\vec{x}_{20} & =\{10,20,5\} \mathrm{km} .
\end{aligned}
$$

The initial positions are $((63 \mathrm{a}),(63 \mathrm{~b}))$ in kilometers $(\mathrm{km})$ and time is in hours (h). The required safe separation distance is

$$
\left|\vec{x}_{1}(t)-\vec{x}_{2}(t)\right|_{\min } \geq \delta=15 \mathrm{~km}
$$

if it is not met then only the vertical velocity of the second aircraft $V_{2 z}$ is to be changed to $V_{2 z}^{\prime}$ by the minimum amount to satisfy the safe separation distance. The safety distances in (42) and (64) are examples, with standard values specified by nautical or aeronautical separation standards. For example, in aeronautics the horizontal separation may be 5 or 10 nautical miles in radar controlled airspace and 50 nautical miles in oceanic airspace and vertical separation may be 2000 or 1000 feet in altitude.

The relative initial position (65a) and the relative velocity (65b) are

$$
\begin{aligned}
\vec{x}_{0} & =\vec{x}_{10}-\vec{x}_{20}=\{-10,-20,5\} \mathrm{km} . \\
\vec{V} & =\vec{V}_{1}-\vec{V}_{2}=\{300,200,-50\} \mathrm{km} / \mathrm{h},
\end{aligned}
$$

and the angle between them is calculated from

$$
\begin{aligned}
\left|\vec{x}_{0}\right| & =22.9 \mathrm{~km}, \\
|\vec{V}| & =364 \mathrm{~km} / \mathrm{h}, \\
\cos \alpha & =\frac{\vec{V} \cdot \vec{x}_{0}}{|\vec{V}|\left|\vec{x}_{0}\right|}=-0.870 .
\end{aligned}
$$

The angle ((67a), (67b)) leads to a distance of closest approach (67c) at the time (67d):

$$
\begin{aligned}
\alpha & =150.43^{\circ}, \\
\sin \alpha & =0.493, \\
D_{\min } & =\left|\vec{x}_{0}\right| \sin \alpha=11.3 \mathrm{~km}, \\
t_{*} & =-\frac{\left|\vec{x}_{0}\right|}{|\vec{V}|} \cos \alpha=0.0547 .
\end{aligned}
$$

The positions of the two aircraft at this time

$$
\begin{aligned}
& \vec{x}_{1}\left(t_{*}\right)=\vec{x}_{10}+\vec{V}_{1} t_{*}=\{49.23,0,10\} \mathrm{km}, \\
& \vec{x}_{2}\left(t_{*}\right)=\vec{x}_{20}+\vec{V}_{2} t_{*}=\{42.82,9.06,7.73\} \mathrm{km}
\end{aligned}
$$

confirm the minimum separation $(67 c) \equiv(69 a)$ :

$$
\begin{aligned}
\left|\vec{x}_{1}\left(t_{*}\right)-\vec{x}_{2}\left(t_{*}\right)\right| & =11.3 \mathrm{~km}=D_{\min }<\delta \\
& =15 \mathrm{~km},
\end{aligned}
$$

which is less than the required safety distance (69b) $\equiv(64)$.

The required separation distance implies that the angle between the relative velocity and initial relative position must satisfy

$$
\begin{aligned}
\sin \alpha^{\prime} & =\frac{\delta}{\left|\vec{x}_{0}\right|}=0.655, \\
\alpha^{\prime} & =139.07^{\circ} \\
\cos \alpha^{\prime} & =-0.7556
\end{aligned}
$$


this will be met by changing only the vertical velocity of the second aircraft from $V_{2 z}=50 \mathrm{~km} / \mathrm{h}$ in $(63 \mathrm{~b})$ to $V_{2 z}^{\prime}$ determined in the sequel. The identity (54) is used again, where (i) the modulus of the relative velocity is

$$
\begin{aligned}
\left|\vec{V}^{\prime}\right|^{2} & =\left|\vec{V}_{1}-\vec{V}_{2}^{\prime}\right|^{2}=300^{2}+200^{2}+\left(V_{2 z}^{\prime}\right)^{2} \\
& =1.3 \times 10^{5}+\left(V_{2 z}^{\prime}\right)^{2} ;
\end{aligned}
$$

(ii) the inner product by the relative position is

$$
\begin{aligned}
\vec{V}^{\prime} \cdot \vec{x}_{0} & =300 x_{0}+200 y_{0}-V_{2 z}^{\prime} z_{0} \\
& =-7 \times 10^{3}-5 V_{2 z}^{\prime} .
\end{aligned}
$$

Substituting ((71a), (71b), (66a)) and (70c) in (54) leads to a quadratic equation for the vertical velocity of the second aircraft:

$$
\left(5 V_{2 z}^{\prime}+7 \times 10^{3}\right)^{2}=299.4\left[1.3 \times 10^{5}+\left(V_{2 z}^{\prime}\right)^{2}\right] .
$$

The binomial $(72 \mathrm{a}) \equiv(72 \mathrm{~b})$

$$
274.4\left(V_{2 z}^{\prime}\right)^{2}-7 \times 10^{4} V_{2 z}^{\prime}-1.0078 \times 10^{7}=0
$$

has roots

$$
V_{2 z}^{\prime}=127.55 \pm 230.21=+357.76,-102.66,
$$

leading to two possible vertical velocities, one climbing and the other descending.

The corresponding relative velocities are

$$
\begin{aligned}
& \left|\vec{V}_{+}^{\prime}\right|=\left|\vec{V}_{1}-\vec{V}_{2+}^{\prime}\right|=507.9 \mathrm{~km} / \mathrm{h}, \\
& \left|\vec{V}_{-}^{\prime}\right|=\left|\vec{V}_{1}-\vec{V}_{2-}^{\prime}\right|=374.9 \mathrm{~km} / \mathrm{h} .
\end{aligned}
$$

The corresponding times of closet approach are

$$
\begin{aligned}
& t_{+}=-\frac{\left|\vec{x}_{0}\right|}{\left|\vec{V}_{+}^{\prime}\right|} \cos \alpha^{\prime}=0.03407 h, \\
& t_{-}=-\frac{\left|\vec{x}_{0}\right|}{\left|\vec{V}_{-}^{\prime}\right|} \cos \alpha^{\prime}=0.04615 h .
\end{aligned}
$$

The relative positions at these times are

$$
\begin{aligned}
\vec{x}_{+} & =\vec{x}_{1}-\vec{x}_{2+}^{\prime}=\vec{x}_{0}+\left(\vec{V}_{1}-\vec{V}_{+}^{\prime}\right) t_{+} \\
& =\{0.221,-13.19,-7.19\}, \\
\vec{x}_{-} & =\vec{x}_{1}-\vec{x}_{2-}^{\prime}=\vec{x}_{0}+\left(\vec{V}_{1}-\vec{V}_{-}^{\prime}\right) t_{-} \\
& =\{3.845,-10.77,9.74\} .
\end{aligned}
$$

Both roots lead to the desired minimum separation distance:

$$
\left|\vec{x}_{-}\right|=15.0 \mathrm{~km}=\delta=\left|\vec{x}_{+}\right|
$$

in different ways, one by descending (73b) and the other by climbing faster (73a). Next is considered a different kind of problem with one curved trajectory and time of closest approach imposed "a priori".

3.3. Minimum Distance between a Curved and a Straight Trajectory. The third example uses SI units for the velocity in meters per second (78b) of an aircraft flying in a circular path of radius (78a) in meters, using time in seconds in the centripetal acceleration (78c) that is small compared with that of gravity:

$$
\begin{aligned}
& R=20 \mathrm{~km}=2 \times 10^{4} \mathrm{~m}, \\
& V=216 \mathrm{~km} / \mathrm{h}=60 \mathrm{~m} / \mathrm{s}, \\
& a=\frac{V^{2}}{R}=0.18 \mathrm{~m} / \mathrm{s}^{2}=0.018 \mathrm{~g} .
\end{aligned}
$$

The trajectory in a Cartesian reference frame with origin at the centre is

$$
\begin{aligned}
& x_{1}(t)=R \cos \left(\frac{V}{R} t\right), \\
& y_{1}(t)=R \sin \left(\frac{V}{R} t\right)
\end{aligned}
$$

where $\Omega=V / R$ is the angular velocity. The second aircraft starts at an initial position (80a) and flies at the same altitude with a constant heading (80b):

$$
\begin{aligned}
\vec{x}_{0} & =\left\{-5 \times 10^{4}, 0\right\} m=\{-L, 0\}, \\
\varphi & =30^{\circ}, \\
t_{*} & =600 s,
\end{aligned}
$$

with constant velocity $u$ such that the time of closest approach is (80c). It is necessary to find the distance of closest approach and the corresponding positions of the two aircraft.

The trajectory of the second aircraft is

$$
\begin{aligned}
& x_{2}(t)=-L+u t \cos \varphi, \\
& y_{2}(t)=u t \sin \varphi .
\end{aligned}
$$

The relative position is

$$
\begin{gathered}
\vec{x}(t)=\vec{x}_{1}(t)-\vec{x}_{2}(t)=\left\{R \cos \left(\frac{V}{R} t\right)+L\right. \\
\left.-u t \cos \varphi, R \sin \left(\frac{V}{R} t\right)-u t \sin \varphi\right\},
\end{gathered}
$$


and its derivative with regard to time

$$
\begin{aligned}
\vec{V} & (t)=\frac{d \vec{x}}{d t} \\
& =\left\{-V \sin \left(\frac{V}{R} t\right)-u \cos \varphi, V \cos \left(\frac{V}{R} t\right)-u \sin \varphi\right\}
\end{aligned}
$$

specifies the relative velocity (83).

The condition of closest approach is $((16 a),(16 b))$ that the relative position (82) and velocity (83) should be orthogonal:

$$
\begin{aligned}
0= & \vec{x}\left(t_{*}\right) \cdot \vec{V}\left(t_{*}\right) \\
= & u^{2} t_{*}-R u \cos \left(\frac{V}{R} t_{*}-\varphi\right)+V u t_{*} \sin \left(\frac{V}{R} t_{*}-\varphi\right) \\
& -L\left[u \cos \varphi+V \sin \left(\frac{V}{R} t_{*}\right)\right] .
\end{aligned}
$$

The time of closest approach (80c) is satisfied if the velocity along the straight path satisfies the quadratic relation:

$$
\begin{aligned}
0= & t_{*} u^{2}+u\left(V t_{*} \sin \psi-R \cos \psi-L \cos \varphi\right) \\
& -L V \sin \phi,
\end{aligned}
$$

where for the present problem ((78a), (78b); (80c), (80b))

$$
\begin{aligned}
& \phi=\frac{V}{R} t_{*}=1.8 \mathrm{rad}=103.13^{\circ}, \\
& \psi=\frac{V}{R} t_{*}-\varphi=73.13^{\circ}
\end{aligned}
$$

the quadratic (85)

$$
0=6 \times 10^{2} u^{2}-1.4655 \times 10^{4} u-2.9216 \times 10^{6}
$$

has roots

$$
u_{ \pm}=12.21 \pm 70.84=+83.05,-58.63 \text {. }
$$

The corresponding positions along the straight path at the time of closest approach are

$$
\begin{aligned}
\vec{x}_{+} & =\left\{-L+u_{+} t_{*} \cos \varphi, u_{+} t_{*} \sin \varphi\right\} \\
& =\left\{-6.85 \times 10^{3}, 2.49 \times 10^{4}\right\}, \\
\vec{x}_{-} & =\left\{-L+u_{-} t_{*} \cos \varphi, u_{-} t_{*} \sin \varphi\right\} \\
& =\left\{-8.05 \times 10^{4},-1.76 \times 10^{4}\right\} ;
\end{aligned}
$$

at the same time the position on the circular path is

$$
\vec{x}=R\{\cos \phi, \sin \phi\}=\left\{-4.54 \times 10^{3}, 1.95 \times 10^{4}\right\} m .
$$

The extrema for the relative distance,

$$
\begin{aligned}
\left|\vec{x}-\vec{x}_{+}\right| & =5.87 \times 10^{3} \mathrm{~m}<8.45 \times 10^{4} \mathrm{~m} \\
& =\left|\vec{x}-\vec{x}_{-}\right|
\end{aligned}
$$

may be local maxima or minima.
To clarify this the acceleration is needed, which is specified by the circular motion alone:

$$
\begin{aligned}
\vec{a} & =\frac{d^{2} \vec{x}}{d t^{2}}=-\frac{V^{2}}{R}\{\cos \phi, \sin \phi\} \\
& =\left\{4.09 \times 10^{-2},-1.75 \times 10^{-1}\right\} \mathrm{m} / \mathrm{s}^{2}
\end{aligned}
$$

Criterion (18b) requires also (83) relative velocities:

$$
\begin{aligned}
\vec{V}_{+} & =\left\{-V \sin \phi-u_{+} \cos \varphi, V \cos \phi-u_{+} \sin \varphi\right\} \\
& =\left\{-1.30 \times 10^{2},-5.52 \times 10\right\} \mathrm{m} / \mathrm{s}, \\
\vec{V}_{-} & =\left\{-V \sin \phi-u_{-} \cos \varphi, V \cos \phi-u_{-} \sin \varphi\right\} \\
& =\{-7.66,1.57 \times 10\} \mathrm{m} / \mathrm{s},
\end{aligned}
$$

through the square of the moduli:

$$
\begin{aligned}
\left|\vec{V}_{+}\right|^{2} & =1.99 \times 10^{4} \mathrm{~m}^{2} / \mathrm{s}^{2}>3.05 \times 10^{2} \mathrm{~m}^{2} / \mathrm{s}^{2} \\
& =\left|\vec{V}_{-}\right|^{2} .
\end{aligned}
$$

Acceleration (92) appears projected on the relative position ((89a), (89b); (90)):

$$
\begin{aligned}
& \vec{x}-\vec{x}_{+}=\left\{2.31 \times 10^{3},-5.40 \times 10^{3}\right\} m, \\
& \vec{x}-\vec{x}_{-}=\left\{7.60 \times 10^{4}, 3.71 \times 10^{4}\right\} \mathrm{m},
\end{aligned}
$$

leading to

$$
\begin{aligned}
& \vec{a} \cdot\left(\vec{x}-\vec{x}_{+}\right)=1.04 \times 10^{3} \mathrm{~m}^{2} / \mathrm{s}^{2} \\
& \vec{a} \cdot\left(\vec{x}-\vec{x}_{-}\right)=-3.38 \times 10^{3} \mathrm{~m}^{2} / \mathrm{s}^{2}
\end{aligned}
$$

Criterion (18b),

$$
\begin{aligned}
& \left|\vec{V}_{+}\right|^{2}+\left(\vec{x}-\vec{x}_{+}\right) \cdot \vec{a}=2.094 \times 10^{4} \mathrm{~m}^{2} / \mathrm{s}^{2}>0 \\
& \left|\vec{V}_{-}\right|^{2}+\left(\vec{x}-\vec{x}_{-}\right) \cdot \vec{a}=-3.075 \times 10^{3} \mathrm{~m}^{2} / \mathrm{s}^{2}<0,
\end{aligned}
$$

shows that the first solution that has positive velocity corresponds to the closest approach and is both a local and a global minimum for the relative distance. The second solution that has negative velocity is a local maximum of the relative distance; it is not an absolute maximum because the latter corresponds to infinite distance.

\section{Discussion}

The problem of conflict avoidance between two vehicles involves, besides a safe separation distance chosen "a priori", 4 vectors, namely, the initial position vectors $\left(\vec{x}_{10}, \vec{x}_{20}\right)$ and constant velocity vectors $\left(\vec{V}_{1}, \vec{V}_{2}\right)$ of each vehicle, leading to $4 \times 2=8$ components in two dimensions and $4 \times$ $3=12$ components in three dimensions. Since a conflict 
is concerned with relative motion, it might be expected that only four variables would be relevant, namely, the relative initial position (9a) and the constant relative velocity (9b). It is remarkable that the distance of closest approach (33) and hence the safety criterion involve only two combinations of these four variables: (i) initial distance (not the direction); (ii) the angle of the relative velocity with the relative initial position (not the modulus of the relative velocity). Thus a conflict can be avoided by (a) steering only one vehicle, so that the condition (36) is met, with no need to change the modulus of the velocity; (b) alternatively the heading that may be retained and conflict avoidance which is implemented by increasing the velocity to race ahead or slowing down or reversing the motion to fall behind. Other less simple combinations are to change the heading and velocity of one or both vehicles. For example, a conflict between aircraft can be resolved by changing speed (acceleration or deceleration) or heading or climb or descend rate for one or both aircraft. The present method applies to all these cases and combinations thereof.

Besides (i) the initial relative distance and (ii) angle of the relative initial position with the relative velocity, the (iii) modulus of the relative velocity also appears in the time of closest approach (28b) $\equiv(37 b)$. The condition of conflict avoidance between two vehicles thus requires that one of them be steered out of a dangerous angular sector for which a collision would occur. In the case of several vehicles a conflict can be avoided if the dangerous angular sectors for each vehicle do not cover all directions of straight travel. If all directions of straight travel lead to conflicts then curved trajectories must be considered. The cases of conflict between vehicles with nonuniform velocity along curved paths are addressed by the first theorem, of which the second is a particular case. The minimum distance between two curves occurs at a point with common normal (Figure 6); this would be the distance of closest approach only if the two vehicles would be at these points at the same time. In that case the relative velocity, that is tangential, would be orthogonal to the relative position, that is along the normal ((16a), (16b)); if the curves have opposite curvatures it would also be locally the point of closest approach ((19a), (19b)). However, in general the vehicles following the two paths will pass by nearest points at different times (Figure 2) and the distance of closest approach will be larger than the minimum distance between the two curves.

In all cases of uniform or nonuniform motion along straight or curved paths it remains true that an extremum of the relative distance (16a) will correspond to a relative velocity orthogonal to the relative position (16b) and that extremum will be the minimum separation distance if the relative acceleration (19a) meets the condition (19b) that may be interpreted as stating that the total acceleration, including the centripetal acceleration, must cause the vehicles to move away from each other. The latter two conditions ((16a), (16b) and (19a), (19b)) are necessary and sufficient to determine the distance of closest approach and apply the criterion of minimum safe separation distance for two vehicles or vehicles with arbitrary trajectories and velocities along them. The minimum is a local minimum for curved trajectories

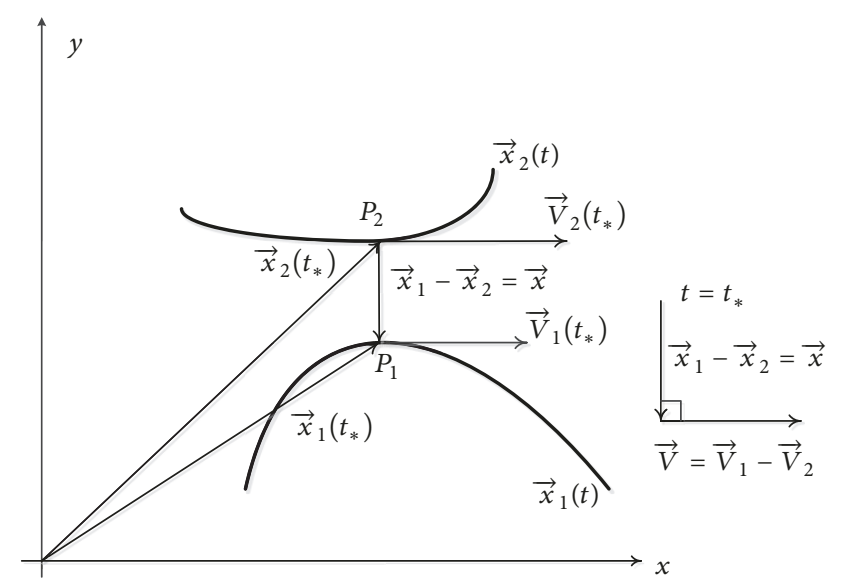

Figure 6: The distance between two paths $\vec{x}_{1}$ and $\vec{x}_{2}$ would be the distance of closest approach between the two trajectories $\vec{x}_{1}(t)$ and $\vec{x}_{2}(t)$ only if the two vehicles were at the closest points $P_{1}$ and $P_{2}$ at the same time $t_{*}$. In that case the relative position $\vec{x} \equiv \vec{x}_{1}-\vec{x}_{2}$ is along the common normal and the relative velocity $\vec{V} \equiv \vec{V}_{1}-\vec{V}_{2}$ is tangential so they are orthogonal $\vec{x} \cdot \vec{V}=0$. This orthogonality condition applies at the time of closest approach (16b) in all cases, even if the vehicles pass at the closest points at different times.

with nonuniform velocity and an absolute minimum for straight trajectories with uniform velocity. In the latter case of straight trajectories with constant velocity (Figure 4) the maximum distance is infinity after an infinite time, and there are no local maxima. In the case of nonuniform motion along curved paths there can exist local minima $D_{*}$ of the relative distance (Figure 3 ) as well as maxima $F_{*}$. The objective of maximizing the relative distance corresponds to (i) relative position orthogonal to the relative velocity, as for any extremum, and (ii) the local acceleration which must overcome the centripetal acceleration to increase the distance. Thus the dotted path in Figure 1 would correspond to a local maximum of the relative distance whereas the solid curve would correspond to a local minimum.

\section{Conclusion}

The conflict detection and resolution $(\mathrm{CDR})$ required that the trajectories of two vehicles lead to a relative distance not less than the safe separation distance (SSD) at all times. This can be ensured if the distance of closest approach (DCA) is not less than the SSD. The DCA has been calculated for two arbitrary trajectories, generally curved and with accelerated motion: in this case there can exist several local minima of the relative distance between two vehicles and the DCA is the smallest of them. In the case of two vehicles with constant velocity the rectilinear trajectories lead to one time of closest approach; the maximum distance is unbounded for large time. The preceding results apply to any type of vehicle (car, ship, and aircraft) and to motion in two or three dimensions. They are illustrated by 2 examples with constant velocities: (i) conflict avoidance between two ships changing the modulus of the velocity of one of them; (ii) conflict avoidance between 
two aircraft changing the climb rate of one of them. A third example involves one straight and one curved trajectory and requires one velocity to be chosen to achieve a time of closest approach chosen "a priori".

\section{List of Symbols}

$\begin{array}{ll}\vec{a}: & \text { Acceleration } \\ D: & \text { Distance between two trajectories } \\ D_{*}: & \text { Distance of closest approach between two } \\ R: & \text { trajectories } \\ t_{*}: & \text { Radius } \\ \vec{u} \cdot \vec{v}: & \text { Time of closest approach } \\ \vec{V}_{1}(t): & \text { Inner product or dot product of two } \\ \vec{V}_{2}(t): & \text { vectors } \vec{u} \text { and } \vec{v} \\ \vec{V}_{:} & \text {Velocity vector of vehicle } 1 \\ \vec{x}_{1}(t): & \text { Relocity vector of vehicle } 2 \\ \vec{x}_{2}(t): & \text { Position vector of vehicle } 1 \\ \vec{x}_{:} & \text {Position vector of vehicle } 2 \\ \vec{x}_{10} \equiv \vec{x}_{1}(0): & \text { Relative position, } \vec{x} \equiv \vec{x}_{1}-\vec{x}_{2} \\ \vec{x}_{20} \equiv \vec{x}_{2}(0): & \text { Initial position of vehicle } 1 \\ \vec{x}_{0} \equiv \vec{x}_{(0):} & \text { Relative initial position, } \vec{x}_{0} \equiv \vec{x}_{10}-\vec{x}_{20} \\ \delta: & \text { Safe separation distance. }\end{array}$

Units

h: Hours

nm: Nautical miles

$\mathrm{km}$ : Kilometers

kt: Knots.

\section{Acronyms}

ATM: Air Traffic Management

CDR: Conflict detection and resolution

DCA: Distance of closest approach

TCA: Time of closest approach

TLS: Target Level of Safety

SSD: Safe separation distance.

\section{Data Availability}

The data used to support the findings of this study are included in the paper.

\section{Conflicts of Interest}

The authors declare that there are no conflicts of interest regarding the publication of this paper.

\section{Acknowledgments}

This work was supported by FCT (Foundation for Science and Technology), through IDMEC (Institute of Mechanical Engineering), under LAETA Pest-OE/EME/LA0022.

\section{References}

[1] J. K. Kuchar and L. C. Yang, "A review of conflict detection and resolution modeling methods," IEEE Transactions on Intelligent Transportation Systems, vol. 1, no. 4, pp. 179-189, 2000.

[2] J. Jansson, Collision Avoidance Theory with Application to Automotive Collision Mitigation, Linköping University, Linköping, Sweden, 2005.

[3] L. M. B. C. Campos and J. M. G. Marques, "On the combination of the gamma and generalized error distribution with application to aircraft flight path deviations," Communications in Statistics-Theory and Methods, vol. 33, no. 10, pp. 2307-2332, 2004.

[4] S. B. McLaughlin, J. M. Hankey, and T. A. Dingus, "A method for evaluating collision avoidance systems using naturalistic driving data," Accident Analysis \& Prevention, vol. 40, no. 1, pp. 8-16, 2008.

[5] A. Tang and A. Yip, "Collision avoidance timing analysis of DSRC-based vehicles," Accident Analysis \& Prevention, vol. 42, no. 1, pp. 182-195, 2010.

[6] S. McPhail, M. Furlong, and M. Pebody, "Low-altitude terrain following and collision avoidance in a flight-class autonomous underwater vehicle," Proceedings of the Institution of Mechanical Engineers, Part M: Journal of Engineering for the Maritime Environment, vol. 224, no. 4, pp. 279-292, 2010.

[7] Z. Lušić and M. Čorić, "Models for estimating the potential number of ship collisions," Journal of Navigation, vol. 68, no. 4 , pp. 735-749, 2015.

[8] P. G. Reich, "Analysis of long-range air traffic systems: separation standards," Journal of Navigation, vol. 19, pp. 88-98, pp. 169186, pp. 331-347, 1966.

[9] L. M. B. C. Campos, "On the probability of collision between aircraft with dissimilar position errors," Journal of Aircraft, vol. 38, pp. 593-599, 2001.

[10] K. Bousson, "Model predictive control approach to global air collision avoidance," Aircraft Engineering and Aerospace Technology, vol. 80, no. 6, pp. 605-612, 2008.

[11] J. F. Shortle, Y. Zhang, and J. Wang, "Statistical characteristics of aircraft arrival tracks," Journal of the Transportation Research Board, no. 2177, pp. 98-104, 2010.

[12] L. F. Vismari and J. B. C. Júnior, "A safety assessment methodology applied to CNS/ATM-based air traffic control system," Reliability Engineering \& System Safety, vol. 96, pp. 727-738, 2011.

[13] A. Narkawicz, C. Muñoz, H. Herencia-Zapana, and G. Hagen, "Formal verification of lateral and temporal safety buffers for state-based conflict detection," Proceedings of the Institution of Mechanical Engineers, Part G: Journal of Aerospace Engineering, vol. 227, no. 9, pp. 1412-1424, 2012.

[14] J. Hu, M. Prandini, and S. Sastry, "Aircraft conflict prediction in the presence of a spatially correlated wind field," IEEE Transactions on Intelligent Transportation Systems, vol. 6, no. 3, pp. 326-340, 2005.

[15] A. L. Visintini, W. Glover, J. Lygeros, and J. Maciejowski, "Monte Carlo optimization for conflict resolution in air traffic control," 
IEEE Transactions on Intelligent Transportation Systems, vol. 7, no. 4, pp. 470-482, 2006.

[16] H. Emami and F. Derakhshan, "A New Method for Conflict Detection and Resolution in Air Traffic Management," in Association for the Advancement of Artificial Intelligence. Report Ws-12-12, pp. 37-40, 2012.

[17] L. M. B. C. Campos and J. M. G. Marques, "On Safety Metrics related to Aircraft Separation," Journal of Navigation, vol. 55, pp. 39-63, 2002.

[18] L. M. B. C. Campos and J. M. G. Marques, "On the probability of collision for crossing aircraft," Aircraft Engineering and Aerospace Technology, vol. 83, no. 5, pp. 306-314, 2011.

[19] L. M. B. C. Campos and J. M. G. Marques, "On the probability of collision between climbing and descending aircraft," Journal of Aircraft, vol. 44, no. 2, pp. 550-557, 2007.

[20] L. M. B. C. Campos and J. M. G. Marques, "On a dimensionless alternative to the ICAO target level of safety," Proceedings of the Institution of Mechanical Engineers, Part G: Journal of Aerospace Engineering, vol. 230, no. 9, pp. 1548-1557, 2016.

[21] J. K. Archibald, S. Member, J. C. Hill, N. A. Jepsen, W. C. Stirling, and R. L. Frost, "A satisficing approach to aircraft conflict resolution," IEEE Transactions on Systems, Man, and Cybernetics, Part C: Applications and Reviews, vol. 38, no. 4, pp. 510-521, 2008.

[22] H. Emami and F. Derakhshan, "An overview on conflict detection and resolution methods in air traffic management using multi agent systems," in Proceedings of the 16th CSI International Symposium on Artificial Intelligence and Signal Processing, AISP 2012, IEEE, May 2012.

[23] H. Emami and F. Derakhshan, "Multi-agent based solution for free flight conflict detection and resolution using particle swarm optimization algorithm," UPB Scientific Bulletin, Series C: Electrical Engineering, vol. 76, no. 3, 2014.

[24] R. Sun, F. Xie, D. Xue, Y. Zhang, and W. Y. Ochieng, "A Novel Rear-End Collision Detection Algorithm Based on GNSS Fusion and ANFIS," Journal of Advanced Transportation, vol. 2017, Article ID 9620831, 10 pages, 2017.

[25] V. Lücken, N. Voss, J. Schreier et al., “Density-based statistical clustering: enabling sidefire ultrasonic traffic sensing in smart cities," Journal of Advanced Transportation, vol. 2018, Article ID 9317291, 15 pages, 2018.

[26] H. Bai, J. Shen, L. Wei, and Z. Feng, "Accelerated lane-changing trajectory planning of automated vehicles with vehicle-tovehicle collaboration," Journal of Advanced Transportation, vol. 2017, Article ID 8132769, 11 pages, 2017.

[27] M. M. Morando, Q. Tian, L. T. Truong, and H. L. Vu, "Studying the safety impact of autonomous vehicles using simulationbased surrogate safety measures," Journal of Advanced Transportation, vol. 2018, Article ID 6135183, 11 pages, 2018. 


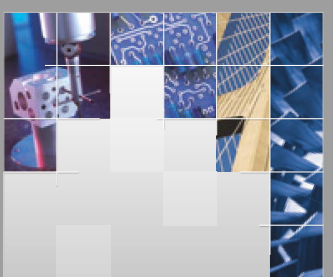

\section{Enfincering}
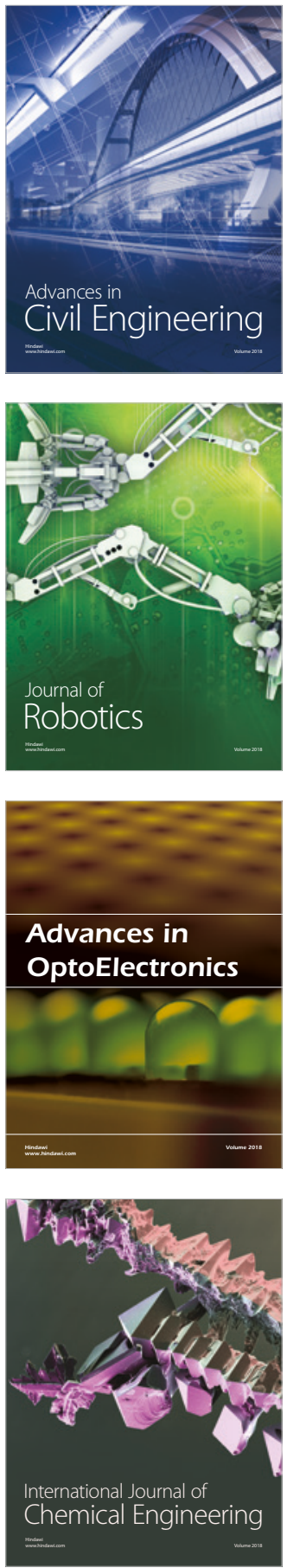

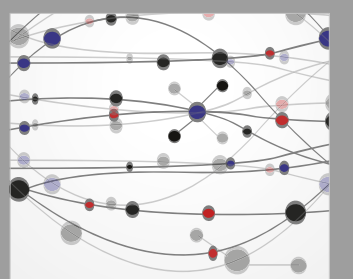

\section{Rotating \\ Machinery}

The Scientific World Journal

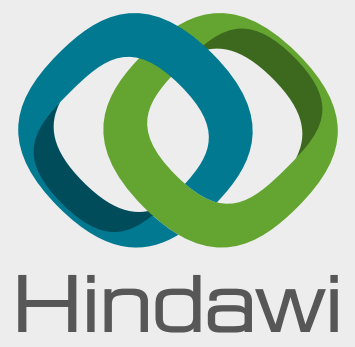

Submit your manuscripts at

www.hindawi.com
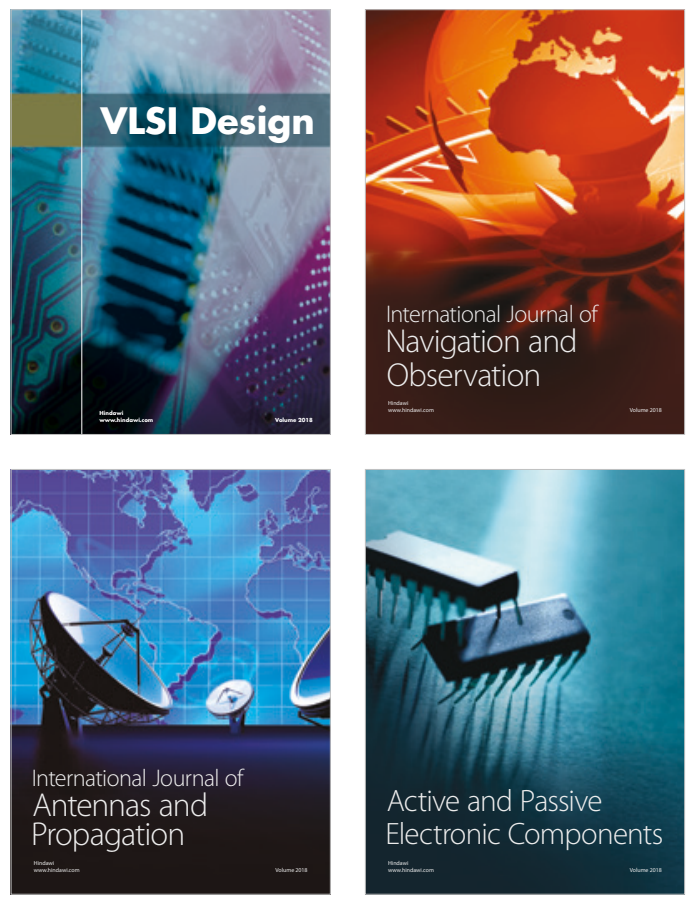
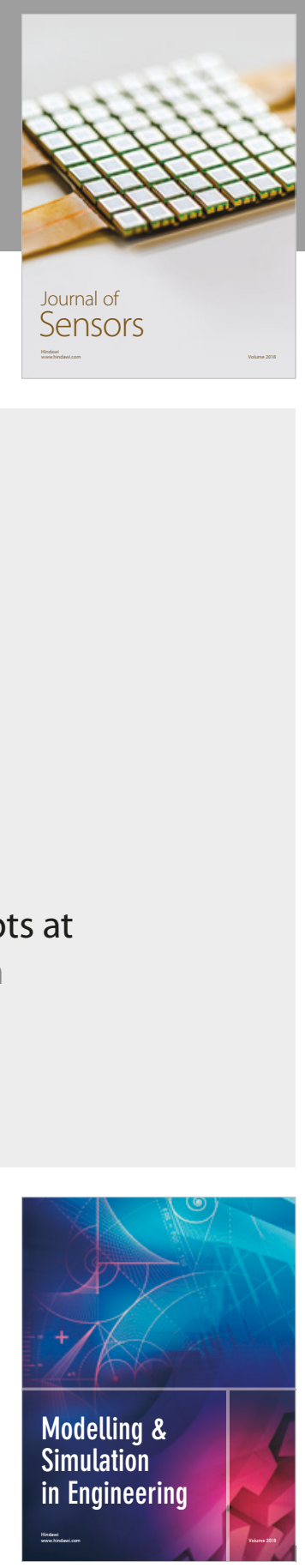

\section{Advances \\ Multimedia}
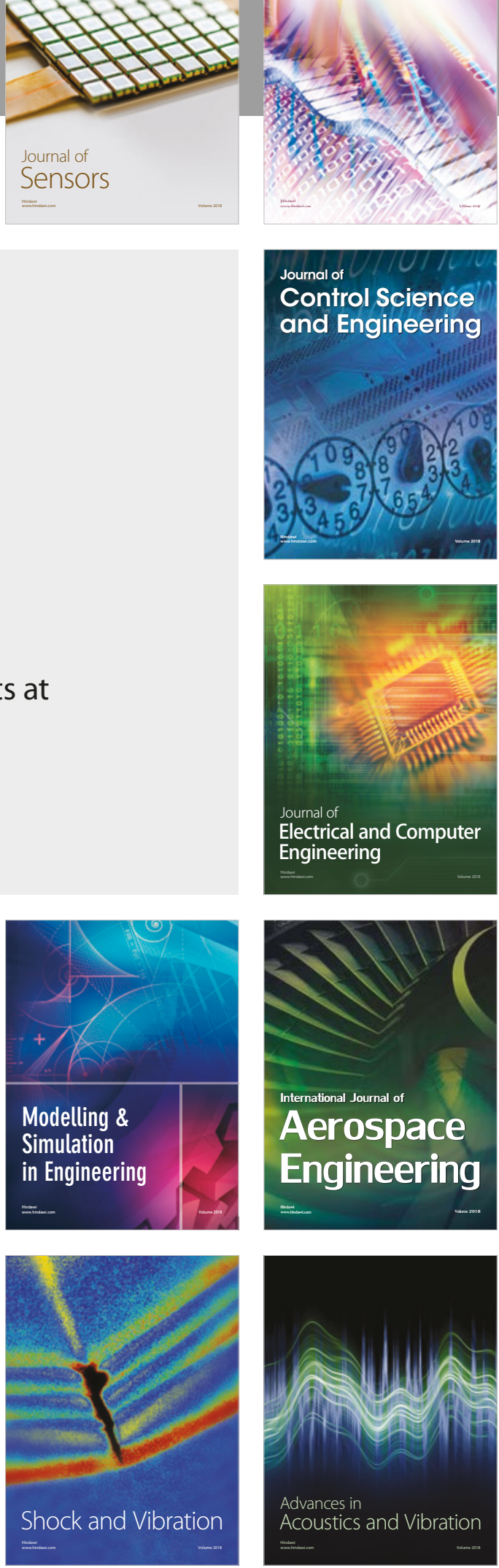